\title{
Electronic-Diary for Recording Headaches, Triggers and Medication Use: Development and Evaluation
}

\author{
Siavash Bandarian-Balooch $\mathrm{PhD}^{1,2}$, Paul R Martin $\mathrm{PhD}^{1,2}$, Brenton McNally $\mathrm{PhD}^{1,2}$, Arissa \\ Brunelli MPsych (Counselling) ${ }^{1,2}$, \& Sharon Mackenzie $\mathrm{PhD}^{1,2}$ \\ ${ }^{1}$ School of Applied Psychology, Griffith University, Queensland, Australia \\ ${ }^{2}$ Menzies Health Institute Queensland, Australia
}

Conflict of Interest Statement: There are no conflicts of interest for any of the authors.

Key words: headache, migraine, triggers, e-diary, reliability, validity.

Sources of financial support: Grants from National Health \& Medical Research Council (ID 1046745) and Menzies Health Institute Queensland.

Acknowledgements: We would like to express our thanks to Mr Mohammad Moezzi at Wexpert Pty Ltd, who developed the e-diaries in accordance with the brief of the authors, and to all the participants in the study.

Abbreviations: e-diary electronic-diary, app application, HDI Headache Disability Inventory, DASS Depression Anxiety Stress Scales, MAQ Measure of Acceptance Questionnaire, TAM Technology Acceptance Model, REGWQ Ryan-Elliot-Gabriel-Welch Q-tests.

Address all correspondence to Siavash Bandarian-Balooch, School of Applied Psychology, Griffith University (Gold Coast Campus), Parklands Drive, Southport, Queensland, 4222, Australia, e-mail: S.bandarian@griffith.edu.au. 


\begin{abstract}
Objective.-To evaluate an e-diary developed for measuring headaches, triggers and medication consumption, in terms of reliability and validity, as well as variables such as ease of use and participant compliance.
\end{abstract}

Background.-For many decades, behavioral treatment of headaches has been evaluated via participants completing paper diaries recording their headaches and associated phenomena. There is some limited evidence supporting the reliability and validity of paper diaries, and criticisms have been offered such as the large amount of effort involved both for participants and researchers. This study evaluates a new e-diary that will operate on virtually any device that can connect to the internet, and yields five of the recommended outcome measures. Methods.-181 participants (146 females, 35 males) were allocated to two groups, e-diaries versus paper diaries, via a disproportionate stratified allocation process. The e-diary group included four subgroups based on the technology available to the participant, and the paper diary group included two subgroups, one parallel to the e-dairies ('short paper'), and one representative of traditional paper diaries ('long paper'). The study commenced with individuals who had self-identified as headache and migraine sufferers attended a screening session which included headache diagnosis. Participants who met selection criteria then completed the Headache Disability Inventory and a measure of quality of life (SF-36) to assess the convergent validity of the diaries, and the Depression Anxiety Stress Scales to assess the discriminant validity of the diaries. They also completed a Measure of Acceptance Questionnaire. Participants then completed the headache diaries over the next 30 days. Finally, participants completed for a second time the questionnaires completed previously. Results.-The five outcome measures - headache frequency, peak intensity, average intensity, duration, and medication usage - were found to have strong test-retest reliability $(r, .68$ to .79), for all three types of diary. These five measures for the e-diaries were shown to have good convergent validity via comparison with scores on the Headache Disability Inventory ( $r$, 
.46 to .55$)$ and SF-36 ( $r,-.35$ to -.49), and divergent validity via comparison with scores on the Depression Anxiety and Stress Scale ( $r, .10$ to .25). The long paper diaries had significantly higher missing data scores $(M=15.20, S D=14.84)$ and more errors in data completion $(M=5.47, S D=3.28)$ than the e-diaries and short paper diaries $(p<.05)$. Also, the long paper diaries were evaluated by the participants as more burdensome and significantly less easy to use than the e-diaries and short paper diaries.

Conclusions.-The e-diaries evaluated in this research would prove a useful tool in clinical trials of behavioral treatment for headaches. 


\section{Electronic-Diary for Recording Headaches, Triggers and Medication use: Development and Evaluation}

Psychologists first became involved in headache research in a significant way in the 1970s with the development of behavioral treatments for headaches. ${ }^{1}$ The efficacy of these treatment programs was typically assessed by the use of headache diaries in which the participants periodically rated their pain, and usually also recorded their use of medication. ${ }^{2}$ The pain scales ranged from 3 points ${ }^{3}$ to 100 points, ${ }^{4}$ and were defined in terms of intensity (e.g., slight, moderate) or impact of the headaches on activities (e.g., a headache that can be ignored at times). The intervals for rating head pain ranged from hourly ${ }^{5}$ to daily (bedtime). ${ }^{6}$ Sometimes additional information was recorded in the diaries such as activities and situations, ${ }^{7}$ impairment/disability from headache, ${ }^{8}$ and pain location. ${ }^{9}$ Headache diaries were typically kept on cards or notebooks that were small enough to be carried around easily. The most commonly used system was the one developed by Budzynski and colleagues which called for hourly ratings on a 6-point scale. ${ }^{5}$

Early research evaluating headache diaries provided some support but also raised issues. For example, Epstein and Abel noted that observation of inpatients who were instructed to record pain on an hourly basis indicated that often clients would not record for several hours, filling in the recordings later from recall. ${ }^{10}$ Collins and Thompson assessed the reliability of recording headache intensity four times a day by implementing three procedures to detect inaccurate recording and reported that $40 \%$ of the subject sample were detected as being noncompliant in their recordings. ${ }^{11}$ However, this study was carried out on a student sample with only $17.7 \%$ experiencing headaches, so perhaps a more positive result would have been obtained with a clinical sample. Blanchard and colleagues investigated the social validity of headache diaries by comparing improvement in headaches following treatment as assessed from the diaries with improvement as assessed by significant others. ${ }^{12}$ The correlation between the measures of change was .44 $(p<.002)$. 
Criticisms can be made of using headache diaries as described above for outcome measures in treatment studies. For example, some of the systems require a lot of effort by the participants, and also by researchers as the data then has to be transcribed for analysis, and the latter has drawbacks in terms of the possibility of making errors as well as utilization of resources. $^{13-15}$

Despite the meagre supportive evidence, and the criticisms of headache diary systems, they have been used for decades in hundreds of studies of behavioral treatment for headaches. In the 'Guidelines for Trials of Behavioral Treatment for Recurrent Headache', ${ }^{16}$ Penzien, Andrasik, and colleagues noted that "Subjective pain ratings of head pain, sampled daily, have come to be regarded as the 'gold standard' in behavioral treatment research” (p. 429). ${ }^{17}$ Reviews of behavioral treatment have focused on different measures derived from headache diaries (e.g., composite indices based on averaging pain ratings that therefore reflect headache frequency, duration and intensity, versus headache frequency/days), but have all used headache diaries for their dependent variables. ${ }^{18,19}$ Presumably, a major reason for this situation, was that for many years there was no obvious alternative to paper-based diaries for frequent recording of pain ratings. This situation changed with the advent of hand-held computers and smartphones.

A more technologically up-to-date method of measuring headache on a daily basis is using computerized daily diaries. ${ }^{20-23}$ A large portion of headache sufferers are internet users and seek information online. ${ }^{23}$ In this context, a range of mobile software/smartphone applications have been developed to assist this population with their migraines and headaches. ${ }^{20}$ However, a review of the literature has shown that computerized headache assessments are restricted to specific measurement devices and smartphone headache applications designed for research are restricted to iPhones. ${ }^{20,24}$

Currently, existing headache diaries often include an excessive number of questions and features, do not include the recommended headache assessment items and do not report 
on participant compliance, accuracy of data gathered or reliability and validity of measurements used. ${ }^{17,24}$ To address this problem, Andrasik and colleagues suggested that all valid headache diaries and measures must contain a primary outcome measure of headache frequency, secondary outcome measures such as duration and intensity of headaches and medication use, and must include no more than 20 items to reduce burden on participants and researchers, and increase compliance. ${ }^{17}$

In this context, a 7-item electronic headache diary system (e-diary) was developed that fitted with the recommendations of Andrasik and colleagues for assessing the outcomes of behavioral treatment for headaches. ${ }^{17}$ The system was designed to work with most devices that can be used for connecting to the internet so that research participants could use their own devices that they were therefore familiar with. As smartphones have the advantage of portability and a wide ownership, an application (app) for smartphones was developed using three different operating systems to cover most smartphones: (i) iOS, the system developed by Apple and used for iPhones and iPads (tablets); (ii) Android, the system developed by Google, and used for smartphones (e.g., Samsung Galaxy) and tablets; and (iii) Windows, the system developed by Microsoft, and used for smartphones (e.g., Sony Erikson) and also Windows computers (laptops and desktops). A website version using HTML was also developed that could be used on any computer (www.enhancediary.com). A paper version of the 7-item e-diary was also developed for participants who were unwilling or unable to use an e-diary.

The current study aimed to test the reliability and validity of the newly developed ediaries. Moreover, the study compared the e-diary and the 7-item paper diary (referred to subsequently as the short paper diary) with a long paper diary used in multiple previous studies. $^{25,26}$ The diaries were compared on percentage of participant non-compliance, timely completion of diaries, accuracy of completed items, and frequency of missing items, 
effectiveness, ease of use and access, usefulness, confidence in using the headache diary, and duration for completion.

The e-diaries and short paper diary recorded the information necessary to generate the primary and secondary outcome measures recommended in the 'Guidelines for Trials of Behavioral Treatment for Recurrent Headache'. ${ }^{16}$ Therefore it was hypothesized that the ediary, short paper diary, and long paper diary would prove to be reliable and valid measures of headaches. It was expected that all diaries would show convergent validity with established measures of headaches, and divergent validity with non-headache specific measures. Based on previous research highlighting differences between e-diaries and paper-based diaries, ${ }^{13-15}$ it was hypothesized that the e-diaries would reveal significantly higher compliance rates than the short and long paper diaries. Moreover, it was hypothesized that the e-diaries would reveal significantly higher accuracy in data entry than the short and long paper diaries. A secondary aim of this study was to examine the success in reducing burden on participants by reducing the number of items on the diaries. ${ }^{15,17}$ Thus, it was expected that the e-diary and the short paper diary would be rated as significantly less burdensome than the long paper diary.

\section{Methods}

\section{Participants}

Initially, 235 participants volunteered for the study. From this group, 54 participants were excluded due to being screened as not suffering from diagnosable headache problems ( $n$ = 36), due to never completing the headache diaries and therefore considered to have chosen to discontinue participation ( $n=6)$, or not attending both testing sessions ( $n=12)$. The remaining 181 participants (146 females and 35 males; age: $M=26.52$ years, range $=18-55$ years, $S D=10.15$ years $)$ reported regular weekly headaches $(M=2.53, S D=1.74)$ and participated in exchange for partial course credit if they were students. Participants from the general community $(n=30)$ were not offered rewards for their participation. All participants were informed of the importance of the study in furthering our knowledge about headache 
severity measurement using paper-based and electronic diaries. Data was collected from April 2014 to October 2016. Participants provided informed consent to a protocol granted institutional ethical approval. Of the sample, 62.1\% were Australian/New Zealander, 6.9\% European, 26.1\% Asian, 2.5\% North American, 1.3\% African, and 1.0\% South American. Participants were recruited via website advertisement and clinically assessed for chronic headache and migraine problems prior to participation. $11.4 \%$ of the sample met criteria for migraine with aura and comorbid tension-type headaches, 13.7\% met criteria for migraine without aura and comorbid tension-type headaches, $11.3 \%$ met criteria for migraine without comorbid headaches, 26.3\% met criteria for tension-type headaches and the remaining $35.4 \%$ of the sample met criteria for a probable migraine or tension-type headache. At baseline, participant reports suggested that thirty three percent of the participants experienced unilateral headache pain, 52\% experienced sensitivity to light, 45\% experienced throbbing headache pain, 42 percent experienced headache episodes between 1-15 days a month, and 58\% experienced headaches more than 15 days per month. Seventy-three percent of the participants diagnosed with one or more headache problems in the current study reported already having been diagnosed and receiving care from their general practitioners or neurologists. To place participant well-being first, the remainder of the diagnosed participants ( $n=54)$ were, during the first assessment session, urged to seek further assistance from their primary health care provider at their earliest convenience.

A disproportionate, partially randomized stratified allocation process was followed, for both the student and community samples. This was due to the large number of subgroups to be compared within a relatively limited sampling pool(i.e. non- iOS phone users). This meant that stratified allocation was utilized but that making the number of participants in each subgroup (stratum) equal was favoured over making them proportionate to the population. Partial randomization meant that, participants were randomly allocated to paper and pencil or e-diary groupings but stratum within those categories were allocated non-randomly to ensure 
equal numbers of participants in each group. None of the participants in the study possessed a smartphone with a Windows operating system, thus all participants in this subgroup were instructed to use the Windows app on their Windows computers (laptops or desktops).

The result of this allocation process was 121 participants in the e-dairy group and 60 participants in the paper diary group, and the following number of participants in each subgroup: (i) iOS ( $n=31)$; (ii) Android ( $n=31)$; (iii) Windows $(n=30)$; (iv) Website (n= 29); (v) Short paper ( $n=30)$; and (vi) Long paper $(n=30)$.

Group membership was independent of gender, $\chi^{2}(5)=6.57, p=.26$.

\section{Measures}

Screening and Clinical Assessment. Assessment of headache diagnoses prior to diary completion and post diary completion was performed by a clinical psychologist specializing in assessment and treatment of headache disorders. Consistent with previous research, ${ }^{27,28}$ clinical assessment was conducted using a structured clinical interview based on the criteria in the International Classification of Headache Disorders, 3rd edition. ${ }^{29}$

E-diaries, Short Paper and Long Paper Diaries. The 7-item e-diary was compared to the 7-item short paper diary and long paper diary. The e-diary contained 7-items that were identical irrespective of the devices and technologies used to complete the e-diary. The short paper diary contained near identical seven items as the e-diary. An example of the short paper diary and e-diary on an Android smartphone (Samsung Galaxy s6) can be seen in Figure 1 and Figure 2, respectively.

Backdated data is particularly concerning in pain research where the accuracy of retrospective reports is affected by current pain experiences. ${ }^{10,11,30,31}$ Item one was included to allow examination of whether the intensity of pain at the moment of diary completion was related to the ratings of peak and average intensity of headaches for the day of completion (i.e., backdated data). The remainder of the items were included in accordance with recommendations by Andrasik and colleagues on best practice for headache measurement. 
Item four, frequency of headaches (attack frequency) was calculated as number of headaches per day. ${ }^{17}$ Items two (peak intensity of headaches), three (average intensity of headaches), and five (duration of headaches) were included as secondary headache outcome measures. Participants' peak and average headache intensity were measured using a scale developed by Budzynski and colleagues where $0=$ No headache, $1=$ A headache of a very low level type which enters awareness only at times when attention is devoted to it, 2 =A headache that can be ignored at times, 3 = A painful headache, but one during which you could continue at your job, 4 = A very severe headache which makes concentration difficult, but during which you could perform tasks of an undemanding nature, $5=$ An intense incapacitating headache. ${ }^{31}$ Item 6 was for recording medication taken for headaches and was included as a secondary non-headache measure. Item seven was included as a non-parametric measure of triggers of headaches and included the most commonly occurring triggers, ${ }^{33}$ such as stress, menstruation, hunger, weather, and lack of sleep. To conduct statistical analyses on the headache trigger data, the triggers were coded and values were provided as outlined in Figure 1 for all diaries. For example, stress was coded as 1, and hunger was coded as 10.

The e-diary also included near-identical calendars as can be seen in Figure 2 (bottom right). The calendar was used as a reminder of days in which the diary was not completed (as displayed in red in Figure 2) and allowed participants to backdate any days they had not completed. The actual and intended date and time of completion scores were recorded in our database. Apart from major software (e.g., iOS, Android, and Windows) and hardware (e.g., laptop and desktop) based differences that are specific to each technology, the e-diary was near identical in navigation, design, colour, feature size ratios, outline, and information. Thus, the example of the e-diary on an Android-based Samsung Galaxy s6 shown in Figure 2 is near identical to the e-diary on other devices.

The e-diary, when completed via iOS, Android, and Windows-based devices, also included an automatic reminder function whereby participants would receive a notice to 
complete their headache diaries 24 hours post non-completion. To reduce interference with the daily lives of participants, the notice would only be sent to the participant a maximum of once every 24 hours and up to a maximum of twice over 48 hours. Thus, after not completing the diary two days in a row, participants would no longer receive notices to complete the diaries. Another feature of the e-diary that was shared on iOS, Android, and Windows devices is that participants only needed to sign-up and login once. After signing up and logging in the first time (through entering a password and their email), the e-diary program would interact with the internal memory of the hard-ware devices used to keep the participants logged in. However, when the e-diary was accessed via the website, the e-diary did not have an automatic reminder function and participants were required to log in to the website diary at each time of diary completion. More computer literate participants reported that they found a way around this by using internet browser functions that automatically input login information when opening the e-diary (Website address www.enhancediary.com). To maximize the utility and ease of use with a variety of technologies, the e-diary was adapted to fit stationary, laptop, smartphone, and electronic pads when it was completed via the website.

The long paper diary required participants to record hourly ratings of headache intensity, medication dosage, medication type, and headache triggers throughout the waking day. Headache intensity was rated using the same 6-point scale as for the e-diaries and short paper diaries.

Compliance and Accuracy Missing data and backdated data were used to examine compliance for each headache diary. Missing data was defined as number of missing items irrespective of inaccurate completion, similar to Palermo and colleagues. ${ }^{14}$ For example, if data for the whole day was not returned, seven missing data scores were recorded. If data was returned but two items not responded to, two missing data scores were recorded. Backdated data was defined as a full days' worth of data that was subjectively reported or objectively recorded to be retrospectively input. For example, if a participant reported that they completed 
the past seven days of diaries in one day, six subjectively reported backdated days were recorded. For the objectively recorded data, the date of actual recorded input (visible on our database) was subtracted from the day recorded data was intended for. We advise caution in interpreting the accuracy findings in the current study as all self-reported headache diary data is at best self-reported estimates of the participants' true headache experience. Thus, in the current study, the term accuracy is differentiated from the term validity by defining accuracy as errors in each diary record. ${ }^{14}$ An example of error is recording " 0 ” on frequency of headaches but still recording a headache duration of more than "0”.

To reduce missing data and inaccurately completed data, all e-diaries included a function whereby data could not be submitted until responses were recorded for all seven items and inaccuracies were remedied. Attempts to submit data without answering all items or providing inaccurate data would result in an automatically generated notice that one or more of the seven items has not been answered correctly and submission was not possible. The unanswered question would be highlighted in red until correctly answered, whereby submission was made possible. With the e-diaries, upon answering the seven items and pressing the submit button, the participant's responses were sent to our database along with the participant's ID, time and date of actual diary completion, and time and date that diary completion was intended for (i.e., when participants were backdating data). The database generated spreadsheets allowing for efficient transfer of data from our database into statistical software for analysis. Data from the paper diaries was entered into statistical software manually.

Diary Evaluation Measures. The 25-item Headache Disability Inventory (HDI) ${ }^{33}$ was used to determine the convergent validity of the headache diaries. The HDI is a reliable and valid measure of disability caused by headaches. ${ }^{34}$ In the current study, the Cronbach's alpha of the total scores for the HDI was .93. To further determine the convergent validity of the headache diaries, the mental health scale of the SF- $36^{34}$ was used as chronic headache and 
migraine have been shown to cause significant reduction in quality of life. ${ }^{36}$ The SF-36 is a reliable and valid measure of quality of life, ${ }^{35}$ and in the current study the Chronbach's alpha for the mental health scale of the SF-36 was .85. Symptoms of depression, anxiety, and stress are common triggers of headache and migraine. ${ }^{2,33}$ The Depression Anxiety Stress Scales 21item version (DASS-21) ${ }^{36}$ was used to examine comparability across groups in terms of depression, anxiety and stress levels, and as a discriminant measure to examine the extent to which the headache diaries could differentiate between headaches and the symptoms of depression, anxiety, and stress. The DASS-21 has been shown to be a reliable and valid measure of severity of depression, anxiety, and stress symptoms. ${ }^{37}$ In the current study, the Cronbach’s alpha for the DASS-21 was .67 for depression, .69 for anxiety, and .64 for stress.

To our knowledge, there is no psychometrically evaluated measure of user acceptance of technologies. Thus, participant evaluations of the diaries were completed using a Measure of Acceptance Questionnaire (MAQ) developed here for evaluation of the diaries (see Table 1). The items of the MAQ were based on the Technology Acceptance Model (TAM), ${ }^{38-41}$ which is the most widely applied information systems theory used to determine usage and acceptance of technology. As can be seen in Table 1, the MAQ included items measuring perceived utility, ease, burden, intention, risk, cost, and confidence in use of the diaries developed in this study. The response options were 10 point Likert scales for all items except one for the one item that required participants to report the average time spent (in minutes) completing the headache dairy each day (see BUR1 in Table 1). Furthermore, as can be seen in Table 1, the MAQ also measured compliance with use of the diaries and compatibility between novel technology (diaries developed here) and current technology (e.g., any existing diaries and technologies participants currently used).

\section{Procedure}

The headache dairies were evaluated in two phases. In the first phase of the study, participants self-identifying as headache and migraine sufferers were invited to participate in 
the study. Subsequent to receiving information about the study and giving consent, participants were invited to partake in a 15-minute screening process. The screening process involved a structured diagnostic interview. Those identified as having a diagnosable headache disorder were required to complete a demographic information questionnaire, the HDI, SF-36, and DASS-21. Subsequently, participants were assigned to groups that involved completing e-diaries or paper diaries, as discussed previously.

All participants were then instructed that they would be required to complete the headache diary once per day, consistently at the same time of the day over the next 30-day period. Participants were strongly encouraged to ensure timely and accurate completion of diaries and reduce need to backdate entries. Participants were also encouraged to review their diary regularly to ensure that they had not omitted data or inaccurately completed data. At this point, follow-up assessment sessions were organized with all participants. Participants in the paper diary subgroups were provided with hard-copies of their diaries concluding the first phase of the study for these participants. Total duration of participation for the paper dairy subgroups in the first phase of the study was 30 minutes.

Participants in the e-diary subgroups were further allocated a password and ID to access their technologies. Participants in the iOS and Android subgroups were instructed to download and install the smartphone applications via the Apple store and Playstore, respectively, under the supervision of the researcher. Participants in the website subgroup were provided the weblink to the e-diary and instructed to login to the website using their preferred technology in the presence of the researcher. Participants in the Windows subgroup were provided a USB with an executable Windows file and were instructed to practice installing the Windows app and login in the presence of the researcher. Next, participants in the e-diary subgroups were provided instructions on how to access the e-diaries, how to navigate the e-diaries, and the various features of the e-diaries. All participants were instructed to complete the headache diary before going to bed after they brush their teeth. This 
concluded the first phase of the study for the participants in the e-diary subgroups. Total duration of participation for the e-diary subgroups in the first phase of the study was 45 minutes.

In the second phase of the study, participants were again required to complete the HDI, SF-36, DASS-21 and MAQ. Subsequently, all participants were asked to subjectively report on the number of days that they had backdated data. All participants were provided with additional information about seeking further assistance to manage their headaches and migraines, concluding the second phase of the study. The total duration of the second phase of the study was 30 minutes. Participant reports of mean time spent completing the diaries on a daily basis between phases was 1.1 minutes ( $S D=0.45$ minutes) per day for the e-diaries, 1.2 minutes $(S D=0.34$ minutes $)$ per day for the short paper diaries, and 7.5 minutes $(S D=2.40$ minutes) per day for the long paper diaries.

\section{Statistical Analyses}

All statistical calculations were conducted using IBM’s SPSS statistics version 23. Reliability was assessed by test-retest analyses. Headache severity is largely state based and has been shown to fluctuate by, for example, hormonal factors such as the female menstrual cycle (e.g., Martin \& MacLeod, 2009). The current sample consisted mainly of females ( $n=$ 146) and hormonal factors were not investigated. Thus, the test-retest reliability coefficients provided here may underestimate the true reliability of the diaries and should be considered estimates only. To examine the validity of the headache diaries, multiple bivariate correlations were conducted between the primary and secondary headache outcome measures derived from the diaries and total scores of the HDI, SF-36, and DASS-21.

There was no difference on the e-diary based on technology used (i.e., the four subgroups - iOS, Android, Windows, or website) in terms of test-retest reliability (range $r=$ .74 - .78) or correlations with convergent and divergent validity measures, all $F^{\prime}$ s $<0.7, p^{\prime}$ s $>$ .14. On this basis, the e-diary data was pooled across the four hardware technologies used. 
Examination of comparability across groups on headache severity including headache intensity, frequency, and duration of headaches, amount of medication used, HDI scores, SF36-MH scores, and DASS-21 scores, using multiple one-way ANOVAS revealed no significant between group differences, $F \mathrm{~s}<2.30$, $p \mathrm{~s}>.05$. The means and standard deviations for the headache diaries, the HDI, SF-36-MH, and DASS-21 are presented in Table 2. Frequency of diagnoses and gender did not significantly differ across groups, $X^{2} \mathrm{~s}<.3 .84, p>$ .58. For the headache diary data, a total of $6.3 \%{ }^{1}$ of the data was missing and was replaced using linear interpolation of scores calculating the average of the score prior to and after the score missing data. The same strategy was used for multiple missing data points. When data points were missing at the start or finish of the diary, the average of two data points after the missing score or two data points prior to the missing score respectively were used. Betweengroups differences were examined using one-way ANOVAs. The dependent variables were frequency of missing data, backdated data, omitted items, errors in data completion, as well as domain scores on the MAQ (see Table 1). The independent variable was diary group, including e-diary, short paper, and long paper. Ryan-Elliot-Gabriel-Welch Q-tests (REGWQ) were used as post hoc analyses to confirm where the differences occurred between the groups. Statistical significance was set at an $\alpha$-level of .05.

\section{Results}

\section{Relationships Between the Variables Monitored by the E-diaries}

The seven variables monitored by the e-diaries are conceptually distinct, but some relationships between them would be anticipated, and how large the relationships would be is an empirical question. Hence, before proceeding with the analyses of reliability and validity, bivariate correlations were calculated between headache frequency, peak and average

\footnotetext{
${ }^{1}$ The long paper diary data had significantly more items than the remainder of the groups. Missing score frequency was therefore standardized for this group to allow for comparison across groups.
} 
headache intensity, average headache duration, and medication use (see Table 3). Table 3 shows significant, moderate, positive correlations between all measures of the e-diaries.

To examine whether current experience of headache pain is related to retrospective reports of pain, multiple bivariate correlation analyses were conducted for the e-diary and short paper diary examining the relationship between headache pain intensity during headache diary completion (item 1) and peak intensity of headaches (item 3). Analyses revealed a significant moderate positive correlation between headache pain intensity during diary completion and peak intensity of headaches for the e-diary group $r=.654, p<.001$, and the short-paper diary group $r=.551, p<.001$.

\section{Reliability of the Headache Diaries}

Test-retest reliability was assessed through bivariate correlation analyses comparing the headache diary means of the first week with the mean of the final week of diary completion. Table 4 shows the test-retest reliability coefficients for the headache diary items, for each diary group. As can be seen in Table 4, analyses revealed highly significant, strong positive correlations between week one and week four headache data for all five variables monitored, for all three types of diary.

Frequency analyses showed that stress (25.9\%), anxiety (21.6\%), depression (19.3\%), anger (4.1\%), dehydration (2.9\%), and lack of sleep (2.8\%) were the most frequently reported

triggers of headaches in the sample. To examine the test-retest reliability of headache triggers, multiple bivariate correlations were used examining the reported headache triggers at the first week of diary completion and final week of diary completion separately for each headache diary. Analyses revealed a significant strong positive correlation between the first week of diary completion and final week of diary completion on headache triggers for the e-diary, $r=$ $.79, p<.001$ and a significant moderate positive correlation for the short paper diary $r=.60, p$ $=.005$ and long paper diary, $r=.49, p=.018$.

\section{Validity of the Headache Diaries}


The convergent and divergent validity of each headache diary were examined separately for each of the diary groups. The correlation between the primary outcome measure of headache frequency, secondary headache outcome measures of peak and average intensity and duration of headaches, and secondary non-headache outcome measure of medication use, and the convergent measures of HDI and SF36-MH, and divergent measure of DASS-21, separately for the e-diary, short paper diary, and long paper diary, are presented in Table 5.

As can be seen in Table 5, bivariate correlations showed that the frequency of headaches on the headache diaries had a significant, moderate, positive correlation with the HDI, and a significant, moderate negative correlation with the SF36-MH, supporting the convergent validity of this primary outcome measure. Table 5 shows a significant, weak, positive correlation between the headache frequency measure of the e-diaries and the DASS21 supporting the divergent validity of this measure. The correlations between the short paper and long paper diaries headache frequency measure and the DASS-21 did not reach significance. The sample size for the short paper and long paper groups was smaller than the e-diary group and so the non-significant findings could be due to less power in the paper groups

For the e-diary, significant, moderate, positive correlations between the secondary headache outcome measures and HDI provided support for convergent validity (see Table 5). This pattern was repeated for the short paper diary average intensity of headaches measure and long paper diary average duration of headaches measure. Weak to moderate, negative, correlations between all secondary headache outcome measures of the e-diary and long paper diary and the SF36-MH also suggested convergent validity. This pattern was replicated for the peak headache intensity measure of the short paper diary. Weak, positive, correlations between all secondary headache outcome measures of the e-diaries and DASS-21 total scores confirmed divergent validity for these measures. The correlations between the short paper and long paper diary secondary headache measures and the DASS-21 did not reach significance. 
For the secondary non-headache outcome measure of medication use, weak to moderate positive correlations with the HDI, and weak to moderate negative correlations with the SF36$\mathrm{MH}$, for each of the headache diaries provided support for convergent validity. Correlations between the medication use measure and DASS-21 did not reach significance for any of the headache diaries supporting divergent validity.

\section{Compliance}

A one-way ANOVA was conducted comparing the six diary subgroups on frequency of missing data (backdated data was not considered missing), corrected for number of items, and revealed a significant difference between subgroups, $F(5,175)=10.50, p<.001, \eta_{p^{2}}=$ .23. As can be seen in Table 6, REGWQ tests revealed that the long paper subgroup had significantly higher missing data scores than all other subgroups. Also, the short paper subgroup had significantly more missing data than the iOS, Android, and Windows subgroups but was not significantly different to the website subgroup. No significant differences were found between the e-diaries on missing data.

To further examine compliance with diary completion, a one-way ANOVA was conducted to compare diary subgroups on subjective reports of backdated data as well as objective records of backdated data. The paper diaries were not included in objective backdated data analyses as objective scores cannot be produced for paper diaries unless participants are being monitored (see Collins \& Thompson $^{41}$ for monitoring of paper diaries). Analyses revealed no significant differences between the subgroups on subjective reports or objective records of backdated data, both $F \mathrm{~s}<1.20$, ps $>.31, \eta_{p}^{2}<.23$.

\section{Accuracy}

A one-way ANOVA comparing all diary subgroups on accuracy of completed data revealed a significant difference between the subgroups, $F(5,175)=65.38, p<.001, \eta_{\mathrm{p}}^{2}=$ 1.87. Post hoc analyses $\left(\alpha^{\prime}=.003\right)$ revealed that the long paper subgroup $(M=5.47, S D=$ 
3.28) had significantly more errors in data completion than the short paper subgroup $(M=$ 3.77, $S D=2.42$ ) and all e-diary subgroups (all e-diary subgroups $M=0, S D=0$ ), all $p$ s < .001 , $d \mathrm{~s}>3.02$. Moreover, the short paper subgroup had significantly more errors in data completion than the e-diary subgroups, all $p s<.001, d s>2.77$. There were no errors in data entry for any of the e-diary subgroups.

\section{Evaluation of Headache Diaries}

Descriptive statistics and internal consistency for each dimension of the MAQ for all diary groups can be seen in Table 7. Multiple one-way ANOVAs were used to compare the diary groups on the individual domains of the MAQ. Analyses revealed no significant difference between the headache diary groups on the domains of utility, risk, cost, and confidence, all Fs $<1.63$, ps $>$.15. However, a significant difference was found between the groups on burden, ease of use, compatibility, and intention to use, all $F s>2.77$, ps $<.02$. As can be seen in Table 8, REGWQ tests revealed that:

- The long paper diary was perceived as significantly more burdensome than the other diaries. No other significant group differences were found.

- The long paper diary was rated as significantly less easy to use than the other diaries. No other significant group differences were found.

- Both the short paper and the long paper diaries were rated as significantly less compatible with the life of the user than the other diary types. The website diary was also rated as significantly less compatible with the life of the user than the Android diary. No other significant group differences were found.

- The long paper diary group rated a significantly lower intention to use the diary than all other groups except the short paper group. No other significant group differences were found.

\section{Conclusion}


The diaries yielded five measures that have been recommended for evaluating outcome in trials of behavioral treatment of headache: ${ }^{17}$ a primary measure of headache (headache frequency); three secondary measures of headache (peak headache intensity, average headache intensity, and average duration of headache); and a secondary nonheadache measure (medication consumption). Preliminary analysis of the e-diary data revealed that the correlations between each of these five variables were significant ( $r=.38$ to .69 ), as expected. However, even the highest correlation indicated that one variable was accounting for less than $50 \%$ of the variance in the other variable, so each variable was adding to the assessment of headaches.

All five recommended outcome measures in the e-diaries, showed good estimates of test-retest reliability, good convergent validity, and good discriminant validity. The same pattern occurred for the paper diaries but some of the correlations did not reach statistical significance. The measure of triggers showed strong estimates of test-retest reliability for the e-diaries $(r=.79)$, and moderate estimates of test-retest reliability for the short paper diaries $(r=.60)$ and long paper diaries $(r=.49)$. These findings support the first hypothesis that the e-diary and short paper diary are reliable and valid measures of headaches. The current study investigated the discriminant validity of the headache diary by examining its correlation with depression, anxiety, and stress symptoms. However, future research could further investigate discriminant validity by examining aspects of psychological distress typically associated with other forms of chronic health conditions. It should be noted that the correlations for test-retest reliability are probably an under estimate, given the following. Menstruation is a commonly reported trigger of both migraine and tension-type headache in females, ${ }^{33}$ and $80.6 \%$ of the sample were females. As data from the first week of recording headaches was compared with data from the fourth week of recording, then for approximately half the female participants, this would have meant that one week was associated with a period and one was not, so that some differences would have been anticipated. 
With respect to compliance with recording requirements, there were more missing data scores in the group using the long paper diary than the e-diary or short paper diary. These findings partially confirm the second hypothesis that higher compliance rates would be observed for the e-diary than the paper diary subgroups. This finding is consistent with previous research showing that electronic headache diaries can significantly reduce missing data compared to paper based headache diaries. ${ }^{13-15}$

In the current study, the reduced missing data for the e-diaries was primarily achieved by participants having a visual reminder about missed days in the form of a calendar. Moreover, the iOS, Android, and Windows smartphone apps automatically generated reminders for participants to complete the headache diary 24 hours after expected entry time. Despite these automatic reminders, there was no difference in missing scores between the smartphone apps subgroups and the website subgroup, which had a visual calendar but no reminder notifications. However, the website subgroup was not significantly different to the short paper diary subgroup in frequency of missing data, suggesting that including visual calendars and reminder notices may have combined effects on reduction of missing data scores. The current study found no difference between any of the subgroups on frequency of backdated data, suggesting that compliance as defined by backdated data is not necessarily resolved by using e-diaries.

The results revealed that all e-diary subgroups had significantly higher accuracy rates than the paper diary subgroups, supporting the third hypothesis. This is consistent with previous research using e-diaries, which has found that inaccurately completed items can be prevented by including software coding in e-diaries which prohibits inaccurate data entry. ${ }^{14}$ Of particular interest, this method of preventing data entry errors was so effective that no inaccurately completed items were detected for any of the e-diary subgroups. However, accuracy does not necessarily suggest validity and until a more valid method of measuring 
headaches becomes readily available, we have to rely on self-reports and interpret findings with caution.

Participants' evaluation of the diaries led to the finding that the long paper diary was viewed as more burdensome and less easy to use than the e-diaries and short paper diaries. The paper diaries were considered less compatible with the life of the user than the e-diaries. The long paper group rated their intention to use the diaries as significantly lower than the ediary group.

Evaluation of the e-diaries revealed that participants rated the long paper diary as more burdensome to use than the e-diaries and short paper diaries, confirming the third hypothesis. This finding is consistent with research that has highlighted the burden that lengthy headache diaries place on participants and researchers alike. ${ }^{15,17}$ Of particular interest, participants did not perceive a difference in burden between the e-diaries and short paper diaries suggesting that in the current study, it was the length of headache diaries and mode of assessment rather than technology used that was associated with participant perception of burden.

Participant evaluation of the headache diaries further revealed that in comparison with the paper diaries, the e-diaries were found to be easier to use and more compatible with participants preferred diary completion method. Moreover, participants reported a stronger intention to continue using the e-diaries than the paper based diaries. Acceptability ratings found here are consistent with previous studies, which have reported high participant acceptability ratings of headache diaries. ${ }^{14,21,43}$ Similar to the current study, Allena and colleagues found that e-diaries were rated as easier to use than paper based diaries. ${ }^{43}$ Previous research has relied on a handful of non-theory based questions in assessment of participant acceptability of electronic and paper based headache measures, and hence there are no comparisons studies available for the current findings on differential group reports on compatibility and intention to use. In the current study, an attempt was made to overcome this limitation by developing the MAQ. 
Hundert et al. (2014) conducted a review of available headache diary apps and encouraged readers to develop apps taking into consideration seven criteria that will significantly improve the scientific expertise and evidence base associated with headache diary apps. ${ }^{44}$ The e-diaries developed here meet each of the seven criteria outline by Hundert et al. (2014). ${ }^{44}$ Our e-diary, was developed by a team of headache experts (criteria 1); the apps underwent psychometric and feasibility testing (criteria 2); the variables measured are clinically relevant (criteria 3) because they were developed for clinical research based on recommendations a review conducted by Andrasik et al. (2005): ${ }^{17}$ participants reported that the e-diaries were easy to use (criteria 4); customized user input is possible (criteria 5) as item 7 allows users to input information of their choosing that is not included in our list of headache triggers; the CSV file produced by the e-diary allows linking of multiple points of information such as duration, severity, and frequency of headaches, associated headache medication usage, and triggers of headaches (criteria 6); the headache data can readily be exported (criteria 7), from our data base via inputting a http address into a web browser that automatically downloads a csv file containing all data input to date. Thus, the app developed in the current study significantly improves on previously developed apps which, at best, met five of the seven criteria developed by Hundert et al. (2014).

In summary, the e-diaries yielded measures that were reliable and valid. In comparisons between the different types of diaries, the e-diaries always emerged as equal or superior to paper diaries. The e-diaries had advantages in terms of compliance and accuracy, and were evaluated more positively by the participants.

The main limitation of the study was that $83.4 \%$ of the sample were drawn from university students studying psychology and the remainder from the community. Hence, it is not known how well these results would generalize to, for example, patients attending generalist or specialist medical clinics. The headaches of participants in the study may have been less severe than those attending clinics, although the study was restricted to individuals 
suffering from at least weekly headaches. The sample was younger and more educated than clinical samples and so it seems plausible that e-diaries would have been more acceptable to this group than clinical samples.

A limitation of the e-diaries is that the cost of developing a system that will work on any smartphone, tablet, laptop, or desktop computer is that it constantly needs updating as manufacturers of these devices bring out new models and change their software. The current team spent approximately 100 hours over an 8 month period developing the many versions of the diary. The system we have developed is currently available for a particular team of researchers because any data collected using the system is automatically stored on a restricted access secure online database. We are working on opening this system to other researchers to use.

In conclusion, we believe that the e-diary system evaluated in this paper would prove a very useful tool in clinical trials of behavioral treatment for headaches. It works as well or better than paper diaries, and relieves researchers of the burden of data entry and corresponding opportunity for errors to be made. 


\section{References}

1. Martin PR. Behavioural research on headaches: Current status and future directions. In: Holroyd KA, Schlote B, Zenz H, eds. Perspectives in Research on Headaches. Toronto: Hogrefe; 1983:204-215.

2. Martin PR. Psychological Management of Chronic Headaches. New York: Guilford Press, 1993.

3. Medina JL, Diamond S. The role of diet in migraine. Headache, 1978;18:31-34.

4. Chesney MA, Shelton JL. A comparison of muscle relaxation and electromyogram biofeedback treatments for muscle contraction headache. Journal of Behavior Therapy and Experimental Psychiatry. 1976;7:221-225.

5. Budzynski TH, Stoyva JM, Adler CS, Mullaney DJ. EMG biofeedback and tension headaches: A controlled outcome study. Psychosomatic Medicine. 1973;35:484-496.

6. Thompson JK, Figueroa JL. Critical issues in the assessment of headache. Progress in Behavior Modification. 1980:15:261-265.

7. Bakal DA, Kaganov JA. A simple method for self-observation of headache frequency, intensity and location. Headache. 1976;16:123-124.

8. Kewman D, Roberts AH. Skin temperature biofeedback and migraine headaches. Biofeedback and Self-Regulation. 1980;5:327-345.

9. Bakal DA. The Psychobiology of Chronic Headache. New York: Springer. 1982.

10. Epstein LH, Abel GG. An analysis of biofeedback training effects for tension headache patients. Behavior Therapy. 1977;8:37-47.

11. Collins FL, Thompson JK. Reliability and standardization in the assessment of selfreported headache pain. Journal of Behavior Assessment. 1979;1:73-86.

12. Blanchard EB, Andrasik F, Neff DF, Jurish SE, O’Keefe DM. Social validation of the headache diary. Behavior Therapy. 1981;12:711-715. 
13. Gwaltney CJ, Shields AL, Shiffman S. Equivalence of electronic and paper-and-pencil administration of patient-reported outcome measures: a meta-analytic review. Value in Health. 2008;11:322-333.

14. Palermo TM, Valenzuela D, Stork PP. A randomized trial of electronic versus paper pain diaries in children: impact on compliance, accuracy, and acceptability. Pain. 2004;107:213-219.

15. Stone AA, Shiffman S, Schwartz JE, Broderick JE, Hufford MR. Patient compliance with paper and electronic diaries. Controlled Clinical Trials. 2003;24:182-199.

16. Penzien DB, Andrasik F, et al. Guidelines for trials of behavioral treatments for recurrent headache, first edition: American Headache Society Behavioural Clinical Trials Workgroup. Headache. 2005;45(S2):S110-132.

17. Andrasik F, Lipchik G, McCrory DC, Wittrock DA. Outcome measurement in behavioral headache research: headache parameters and psychosocial outcomes. Headache. 2005;45:429-437.

18. Rains JC, Penzien DB, McCrory DC, Gray RN. Behavioral headache treatment: history, review of the empirical literature, and methodological critique. Headache. 2005;45(S2):S92-S109.

19. Sullivan A, Cousins S, Ridsdale L. Psychological interventions for migraine: A systematic review. Journal of Neurology. 2016.

20. Liu C, Holroyd KA, Zhu Q, Shen K, Zhou W. Design and implementation of a behavioral migraine management iPhone app for adolescents with migraine. In World of Wireless Mobile and Multimedia Networks (WoWMoM), 2010 IEEE International Symposium on a (pp. 1-6). IEEE.

21. Sorbi M, Mak S, Houtveen J, Kleiboer A, van Doornen L. Mobile Web-based monitoring and coaching: feasibility in chronic migraine. Journal of Medical Internet Research. 2007;9:e38. 
22. Stone AA, Shiffman S. Capturing momentary, self-report data: A proposal for reporting guidelines. Annals of Behavioral Medicine. 2002; 24:236-243.

23. Ybarra ML, Suman M. Help seeking behavior and the Internet: A national survey. International Journal of Medical Informatics. 2006;75:29-41.

24. Stinson JN, Huguet A, McGrath P, Rosenbloom B, Soobiah C, White M, Coburn G. A qualitative review of the psychometric properties and feasibility of electronic headache diaries for children and adults: where we are and where we need to go. Pain Research and Management. 2013;18:142-152.

25. Martin PR, Forsyth MR, Reece J. Cognitive-behavioral therapy versus temporal pulse amplitude biofeedback training for recurrent headache. Behavior Therapy. 2007;38:350-363.

26. Martin PR, Nathan PR, Milech D, van Keppel M. Cognitive therapy vs. selfmanagement training in the treatment of chronic headache. British Journal of Clinical Psychology. 1989;28:347-361.

27. Martin PR, Reece J, Callan M, MacLeod C, Kaur A, Gregg K, Goadsby PJ. Behavioral management of the triggers of recurrent headache: a randomized controlled trial. Behaviour Research and Therapy. 2014;61:1-11.

28. Martin VT, Fanning KM, Serrano D, Buse DC, Reed ML, Bernstein JA, Lipton RB. Chronic rhinitis and its association with headache frequency and disability in persons with migraine: Results of the American Migraine Prevalence and Prevention (AMPP) Study. Cephalalgia. 2014;34:336-348.

29. Headache Classification Committee of the International Headache Society (IHS): The international classification of headache disorders, 3rd edition (beta version). Cephalalgia. 2013;33:627-808.

30. Eich E, Reeves JL, Jaeger B, Graff-Radford SB. Memory for pain: Relation between past and present pain intensity. Pain. 1985;23:375-379. 
31. Wittrock DA, Foraker S. Tension-type headache and stressful events: The role of selective memory in the recall of stressful events. Headache. 2001;41:482-493.

32. Budzynski T, Stoyva J, Adler C. Feedback-induced muscle relaxation: Application to tension headache. Journal of Behavior Therapy and Experimental Psychiatry. 1970;1:205-211.

33. Martin PR, MacLeod C. Behavioral management of headache triggers: Avoidance of triggers is an inadequate strategy. Clinical Psychology Review. 2009;29: 483-495.

34. Jacobson GP, Ramadan NM, Aggarwal SK, Newman CW. The Henry Ford Hospital Headache Disability Inventory (HDI). Neurology. 1994;44:837-842.

35. Ware Jr, JE, Gandek B. The SF-36 Health Survey: development and use in mental health research and the IQOLA Project. International Journal of Mental Health. 1994;23:49-73.

36. Steiner TL, Birbeck GL, Jensen RH, Katsavara Z, Stovner LJ, Martelletti P. Headache disorders are the third cause of disability worldwide. The Journal of Headache \& Pain. 2015;6:429-440.

37. Henry JD, Crawford JR. The short-form version of the Depression Anxiety Stress Scales (DASS-21): Construct validity and normative data in a large non-clinical sample. British Journal of Clinical Psychology. 2005;44:227-239.

38. Davis FD, Bagozzi RP, Warshaw PR. User acceptance of computer technology: a comparison of two theoretical models. Management Science. 1989;35:982-1003.

39. Venkatesh V. Determinants of perceived ease of use: Integrating control, intrinsic motivation, and emotion into the technology acceptance model. Information Systems Research. 2000;11:342-365.

40. Venkatesh V, Davis FD. A theoretical extension of the technology acceptance model: Four longitudinal field studies. Management Science. 2000;46:186-204. 
41. Venkatesh V, Morris MG, Davis GB, Davis FD. (2003). User acceptance of information technology: Toward a unified view. MIS Quarterly. 2003;425-478.

42. Collins FL, Thompson JK. Assessing self-report of pain: a Comparison of two recording procedures. Journal of Behavioral Assessment. 1980;2:55-63.

43. Allena M, Cuzzoni MG, Tassorelli C, Nappi G, Antonaci F. An electronic diary on a palm device for headache monitoring: a preliminary experience. The Journal of Headache and Pain. 2012;13:537-541.

44. Hundert AS, Huguet A, McGrath PJ, Stinson NJ, Wheaton M. Commercially Available Mobile Phone Headache Diary Apps: A Systematic Review. Journal of Medical Internet Research. 2014:3:e36. 
Tables

Table 1. Items of the Measure of Acceptance Questionnaire (MAQ; 39 items) used to Measure

\section{Participant Acceptance and Usage of the E-Diaries and Paper Diaries}

The headache diaries were useful for helping me detect headache triggers

The headache diaries were useful for increasing my awareness of my difficulties

The headache diaries were useful for improving how I deal with my difficulties

The headache diaries would be useful for helping me engage in treatment for my difficulties

Note. UTI = Utility, EOU = Ease of use, BUR = Burden, INT = Intention to use, COMP = Compatibility, CONF $=$ confidence, $*=$ Reverse-scored item; Response options for UTI1-EOU4 were $1=$ I do not agree, 5 $=\mathrm{I}$ somewhat agree, $10 \mathrm{I}$ fully agree. Response options EOU5-EOU8 were $1=$ not at all easy, $5=$ somewhat easy, 10 = very easy. 
Table 2. Means and Standard Deviations for the Headache Diary, HDI, SF36-MH, and DASS-21 for the Three Headache Diary Groups

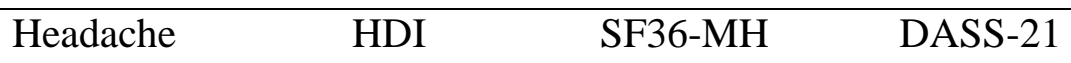

Diary

\begin{tabular}{lcccccccc} 
Diary Type & $M$ & $S D$ & $M$ & $S D$ & $M$ & $S D$ & $M$ & $S D$ \\
\hline E-diary & 2.03 & 1.22 & 36.78 & 20.29 & 27.16 & 17.99 & 33.43 & 22.67 \\
Short paper diary & 1.94 & 1.10 & 37.72 & 19.71 & 29.58 & 22.18 & 17.37 & 13.62 \\
Long paper diary & 2.29 & 1.28 & 38.42 & 19.58 & 23.53 & 20.33 & 21.52 & 13.81
\end{tabular}

Note. HDI = Headache Disability Inventory total scores, SF36-MH = SF36 mental health domain. DASS-21 = Depression Anxiety Stress Scales 21-item total scores. 
Table 3. Bivariate Correlations Between Headache Frequency, Peak Headache Intensity, Average

Headache Intensity, Duration of Headache, and Medication Use for the E-diary $(n=121)$

\begin{tabular}{llllll}
\hline Measure & Frequency & Peak & Average & Duration & Medication \\
& \multicolumn{7}{c}{ Intensity } & Intensity \\
& - & $.44^{* *}$ & $.51^{* *}$ & $.38^{* *}$ & $.59^{* *}$ \\
Frequency & - & $.69^{* *}$ & $.59^{* *}$ & $.58^{* *}$ \\
Peak intensity & & - & $.51^{* *}$ & $.60^{* *}$ \\
Average intensity & & & - & $.67^{* *}$ \\
Duration & & & & \\
Medication & & & &
\end{tabular}

Note. Frequency = frequency of headaches; Peak Intensity = peak daily intensity of headaches; Average Intensity = average daily intensity of headaches; Duration = daily duration of headaches in hours; Medication $=$ number of pills taken per day to manage headaches. $* *=p<.001$ 
Table 4. Estimated Test-Retest Reliability of Headache Frequency, Peak Intensity, Average Intensity, Duration, and Medication Use for Each of the Headache Diaries

Diary Group
Test-retest reliability

Coefficient $(r)$

\begin{tabular}{lccccc}
\hline & & Peak & Average & & \\
& Frequency & & & Duration & Medication \\
E-diary $(n=121)$ & $.79 * *$ & $.73^{* *}$ & $.76^{* *}$ & $.79^{* *}$ & $.79 * *$ \\
Short paper diary $(n=30)$ & $.75^{* *}$ & $.71^{* *}$ & $.73^{* *}$ & $.73^{* *}$ & $.76^{* *}$ \\
Long paper diary $(n=30)$ & $.69^{* *}$ & $.68^{* *}$ & $.70^{* *}$ & $.69^{* *}$ & $.73^{* *}$
\end{tabular}

Note. ${ }^{* *}=p<.001$. Frequency $=$ frequency of headaches; Peak Intensity $=$ peak daily intensity of headaches; Average Intensity = average daily intensity of headaches; Duration = daily duration of headaches in hours; Medication = number of pills taken per day to manage headaches. 
Table 5. Bivariate Correlations Between Each of the Headache Diaries and the

Convergent Validity Measures of HDI and SF36-MH, and the Divergent Validity

Measure of DASS-21

\begin{tabular}{|c|c|c|c|}
\hline Diary Type and Measure & HDI & SF36-MH & DASS-21 \\
\hline \multicolumn{4}{|l|}{ E-diary $(n=121)$} \\
\hline Headache frequency & $.55 * *$ & $-.44 * *$ & $.10^{*}$ \\
\hline Peak headache intensity & $.46^{* *}$ & $-.49 * *$ & $.18^{*}$ \\
\hline Average headache intensity & $.49 * *$ & $-.38 * *$ & $.17^{*}$ \\
\hline Average duration of headache & $.51 * *$ & $-.39 * *$ & $.25 * *$ \\
\hline Medication use & $.65 * *$ & $-.35 * *$ & $.17^{*}$ \\
\hline \multicolumn{4}{|l|}{ Short paper diary $(n=30)$} \\
\hline Headache frequency & $.52 * *$ & $-.66 *$ & .01 \\
\hline Peak headache intensity & .26 & $-.30 *$ & .03 \\
\hline Average headache intensity & $.44^{*}$ & -.35 & .18 \\
\hline Average duration of headache & .32 & -.26 & .29 \\
\hline Medication use & $.58 * *$ & $-.43 * *$ & .13 \\
\hline \multicolumn{4}{|l|}{ Long paper diary $(n=30)$} \\
\hline Headache frequency & $.42^{*}$ & $-.41 *$ & .05 \\
\hline Peak headache intensity & .30 & $-.44 *$ & .04 \\
\hline Average headache intensity & .32 & $-.39 *$ & .03 \\
\hline Average duration of headache & $.61 * *$ & $-.57 * *$ & .28 \\
\hline Medication use & $.66 * *$ & $-.38 *$ & .17 \\
\hline
\end{tabular}

Note. ${ }^{* *}=p<.001, *=p<.05$. HDI $=$ Headache Disability Inventory total scores, SF36$\mathrm{MH}=$ SF36 mental health domain. DASS-21 = Depression Anxiety Stress Scales 21-item total scores. 
Table 6. Results of REGWQ Tests for Differences in Missing Data Between Diary

\section{Groups}

\begin{tabular}{lccc}
\hline Diary Group & $M$ & $S D$ & $n$ \\
\hline Long paper diary & $27.36^{\mathrm{a}}$ & 26.71 & 30 \\
Short paper diary & $17.82^{\mathrm{b}}$ & 15.75 & 30 \\
Website & $10.98^{\mathrm{bc}}$ & 10.19 & 29 \\
iOS & $8.24^{\mathrm{c}}$ & 6.53 & 31 \\
Android & $6.16^{\mathrm{c}}$ & 4.39 & 31 \\
Windows & $6.61^{\mathrm{c}}$ & 4.56 & 30
\end{tabular}

Note. Means sharing a letter in their superscript are not significantly different at the .05 level according to a REGWQ test. 
Table 7. Descriptive Statistics and Internal Consistency for MAQ Variables

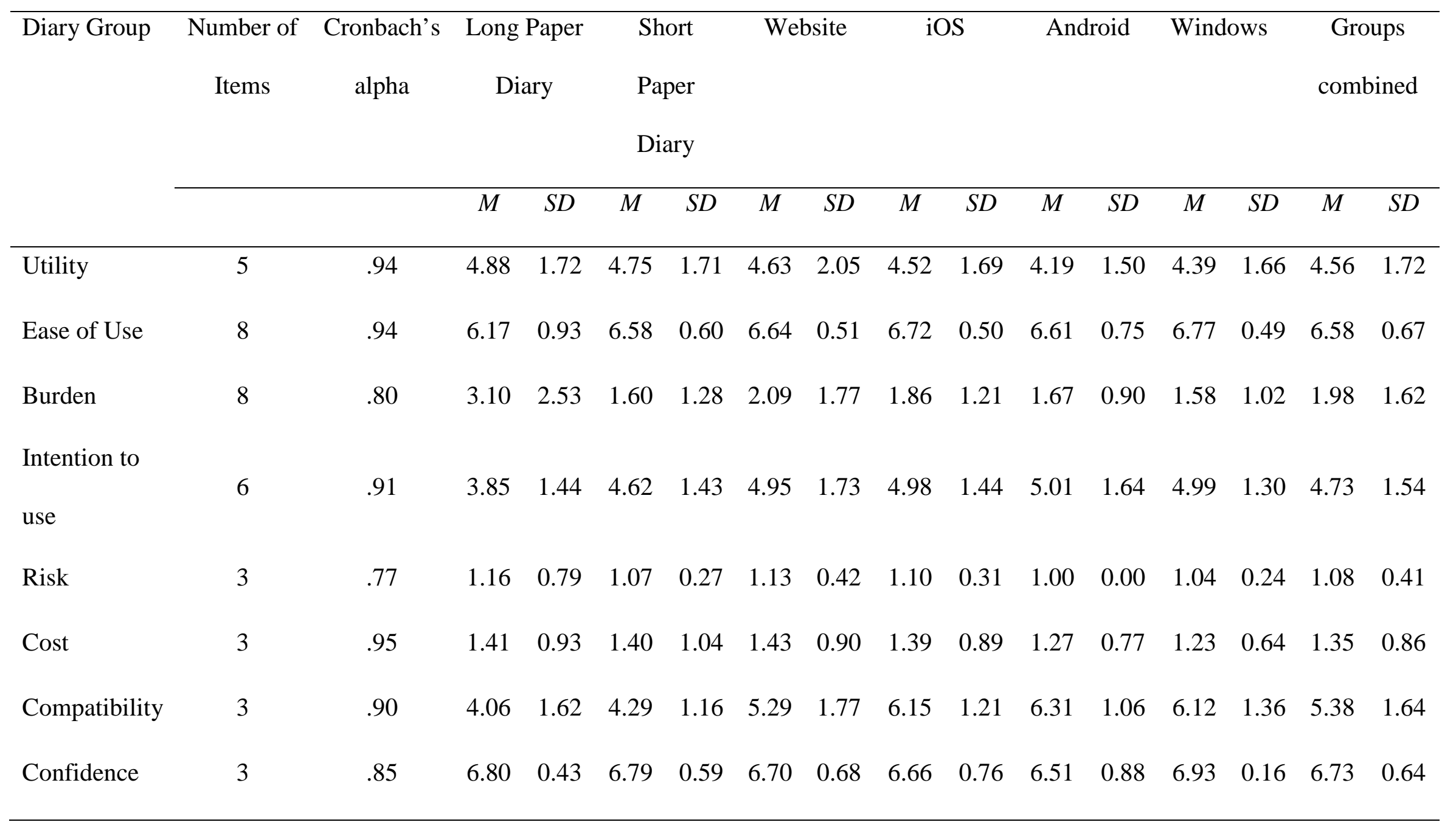


Table 8. Results of REGWQ tests for Differences Between Diary Groups on MAQ domains of Burden, Ease of Use, Compatibility, and Intention to Use

\begin{tabular}{|c|c|c|c|c|c|c|c|c|c|}
\hline \multirow{2}{*}{ Diary Group } & \multicolumn{2}{|c|}{ Burden } & \multicolumn{2}{|c|}{ Ease of Use } & \multicolumn{2}{|c|}{ Compatibility } & \multicolumn{2}{|c|}{ Intention to Use } & \multirow{2}{*}{$n$} \\
\hline & $M$ & $S D$ & $M$ & $S D$ & $M$ & $S D$ & $M$ & $S D$ & \\
\hline Long paper diary & $3.10^{\mathrm{a}}$ & 2.53 & $6.17^{\mathrm{a}}$ & 0.93 & $4.06^{\mathrm{a}}$ & 1.62 & $3.85^{\mathrm{a}}$ & 1.44 & 30 \\
\hline Short paper diary & $1.60^{\mathrm{b}}$ & 1.28 & $6.58^{\mathrm{b}}$ & 0.60 & $4.29^{\mathrm{a}}$ & 1.16 & $4.62^{\mathrm{ab}}$ & 1.43 & 30 \\
\hline Website & $2.09^{b}$ & 1.77 & $6.64^{\mathrm{b}}$ & 0.51 & $5.29^{b}$ & 1.77 & $4.95^{b}$ & 1.73 & 29 \\
\hline $\mathrm{iOS}$ & $1.86^{\mathrm{b}}$ & 1.21 & $6.72^{\mathrm{b}}$ & 0.50 & $6.15^{\mathrm{bc}}$ & 1.21 & $4.98^{\mathrm{b}}$ & 1.44 & 31 \\
\hline Android & $1.67^{\mathrm{b}}$ & 0.90 & $6.61^{\mathrm{b}}$ & 0.75 & $6.31^{\mathrm{c}}$ & 1.06 & $5.01^{b}$ & 1.64 & 31 \\
\hline Windows & $1.58^{\mathrm{b}}$ & 1.02 & $6.77^{\mathrm{b}}$ & 0.49 & $6.12^{\mathrm{bc}}$ & 1.36 & $4.99^{\mathrm{b}}$ & 1.30 & 30 \\
\hline
\end{tabular}

Note. Means sharing a letter in their superscript are not significantly different at the .05 level according to a REGWQ test. 


\section{Figures}

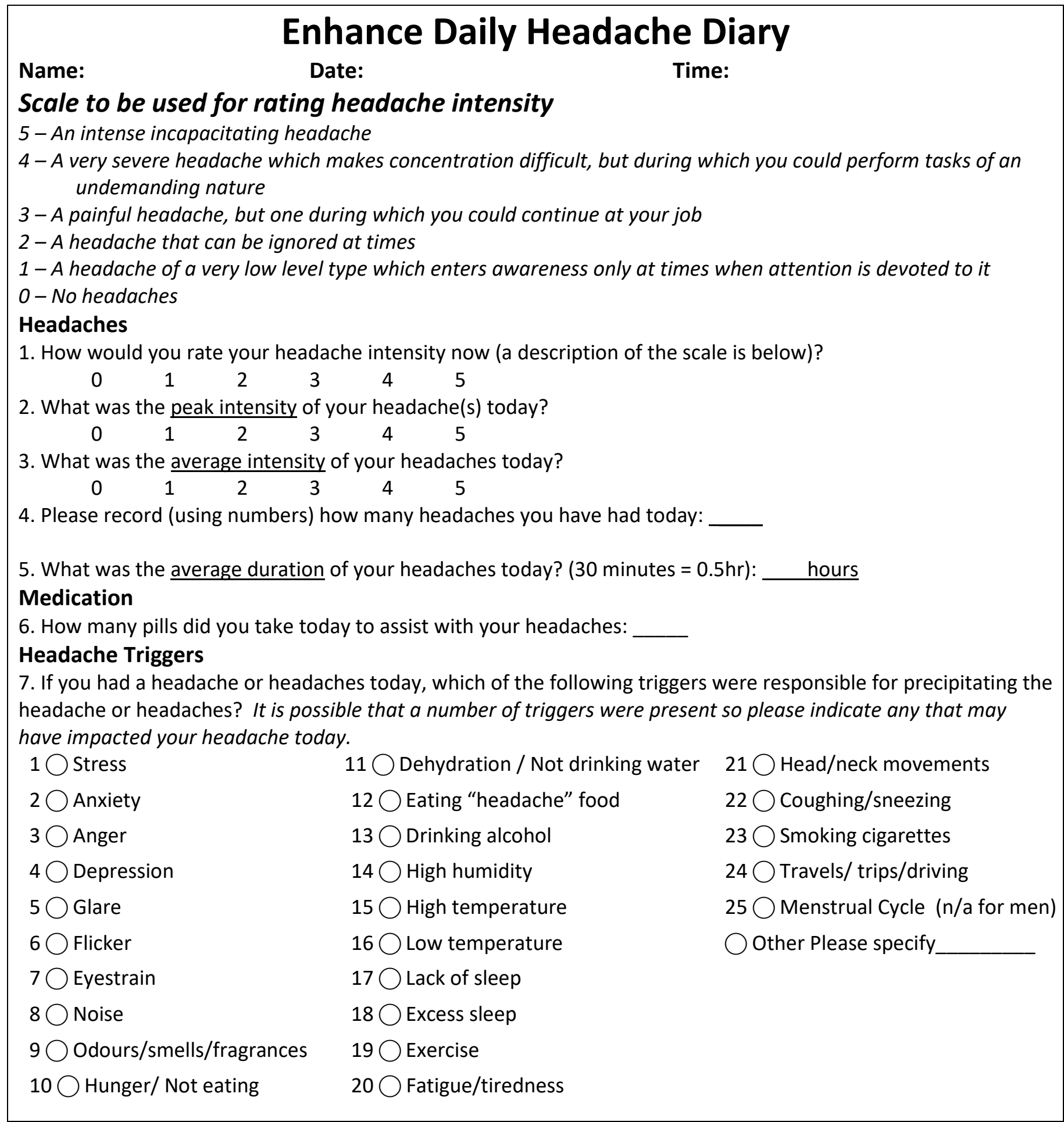

Figure 1. The paper version of the 7-item diary 

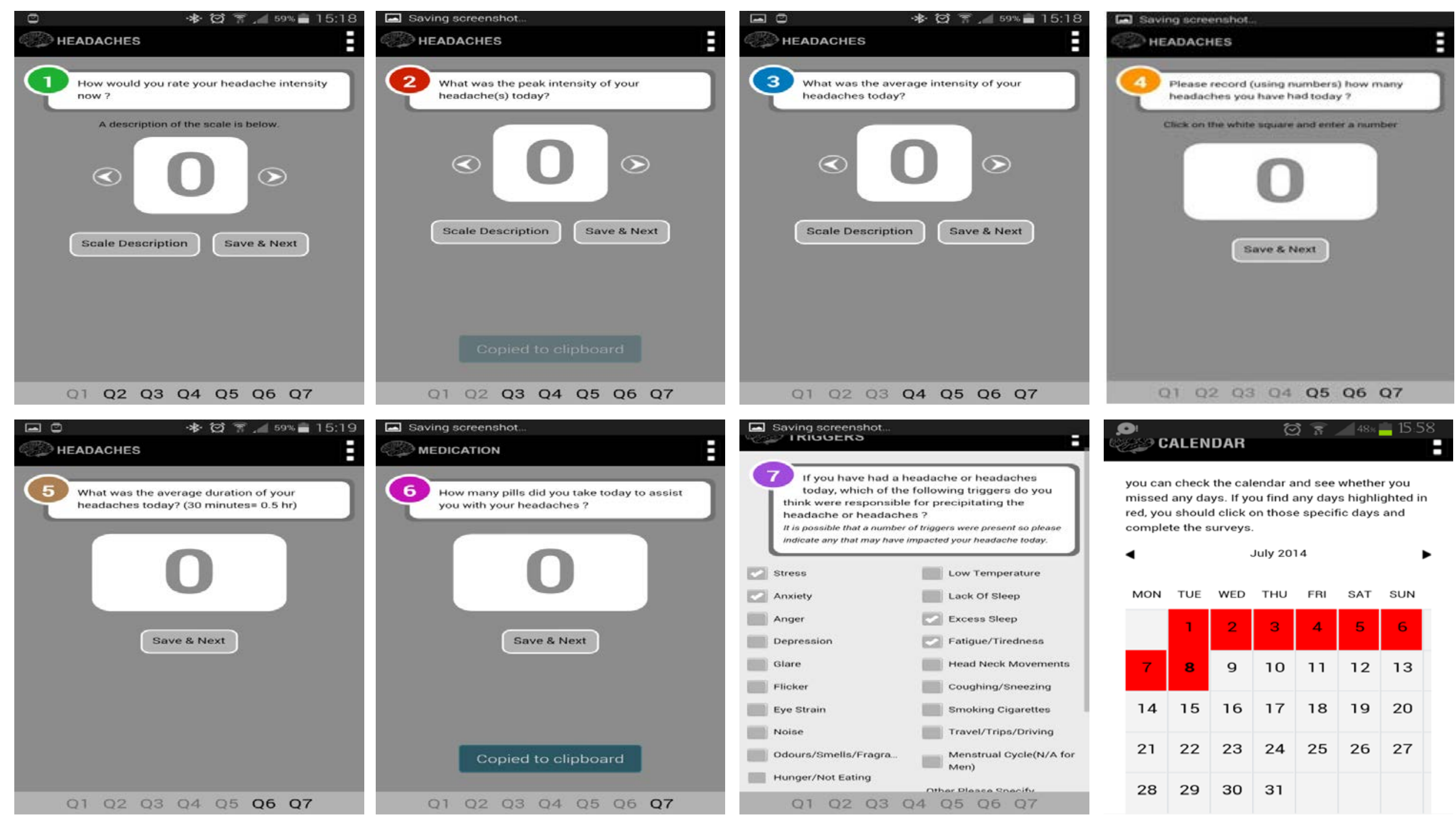

you can check the calendar and see whether you missed any days. If you find any days highlighted in red, you should click on those specific days and complete the surveys.

MON TUE WED THU FRI SAT SUN

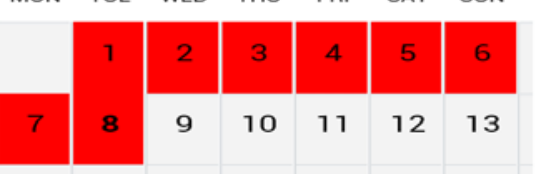

\begin{tabular}{l|l|l|l|l|l|l}
14 & 15 & 16 & 17 & 18 & 19 & 20
\end{tabular}

\begin{tabular}{l|l|l|l|l|l|l}
21 & 22 & 23 & 24 & 25 & 26 & 27
\end{tabular}

\begin{tabular}{l|l|l|l}
28 & 29 & 30 & 31
\end{tabular}

Figure 2. Android Smartphone electronic diary screenshots. The design, colour, wording, items, response options and response methods were identical between the identical between the electronic diary versions 